\title{
The successful retrieval of a broken guide wire from the diagonal branch of the left anterior descending coronary artery complicated by partial stent rolling
}

\author{
Rafał Januszek, Stanisław Bartuś, Artur Dziewierz, Dariusz Dudek \\ $2^{\text {nd }}$ Department of Cardiology, University Hospital, Krakow, Poland
}

Adv Interv Cardiol 2016; 12, 2 (44): 166-170 DOI: 10.5114/aic.2016.59368

\section{Introduction}

Percutaneous coronary interventions (PCls) are relatively safe, and the complication rate is low in comparison to cardiac surgery revascularization. The incidence of broken or retained $\mathrm{PCl}$ equipment is about $0.1-0.8 \%$ [1]. We report a successful retrieval of a fractured guide wire by using a percutaneous catheter Amplatz GooseNeck Snare and Microsnare Kit (Covidien, Plymouth, USA), which was complicated by stent deformation in the form of rolling up its distal part after retracting a piece of the guide wire. This artificial damage to the implanted stent was initially ineffectively treated with balloon angioplasty and finally successfully with double drug-eluting stent (DES) deployment.

\section{Case report}

The patient was a 60-year-old man, with effort angina class II, admitted to our hospital for elective PCl of the left main coronary artery (LMCA) and the left anterior descending coronary artery (LAD). The patient was a heavy smoker for more than 40 years, with arterial hypertension, heart failure, hypercholesterolemia, obesity, and osteoarthritis of the spine. The patient had been diagnosed with myocardial infarction 2 weeks before, and primary $\mathrm{PCl}$ of the right coronary artery with DES implantation was performed previously. His prior myocardial infarction was complicated by non-sustained ventricular tachycardia including torsades de pointes. Angiography revealed significant lesion in the distal LMCA and proximal LAD, which were confirmed by intravascular ultrasound (IVUS) (Figures 1 A, 2 A). The patient was qualified for the second stage of endovascular revascularization by heart team council. At admission the patient was stable, without clinical symptoms, cardiopulmonary and respiratory aligned. A transthoracic echocar- diogram showed a normal left ventricular fraction (50\%) with hypokinesis of the posterior wall.

We performed $\mathrm{PCl}$ of LMCA/LAD using the femoral approach. The 7 Fr extra back-up 3.5 (Medtronic, Inc. Minneapolis, Minnesota, USA) guide catheter was introduced. Then BMW Elite and Universal II (Abbott Vascular, Santa Clara, California, USA) guide wires were introduced into the LAD, diagonal branch (Dg) 1 and Dg2. We implanted directly DES Xience PRO ${ }^{\times}$(Abbott Vascular, Santa Clara, California, USA) $3.0 \times 28 \mathrm{~mm}$ in the LAD (Figure $1 \mathrm{~B}$ ). Afterwards, post-dilatation was carried out with the non-compliant balloon catheter $3.5 \times 12 \mathrm{~mm}$ EmergeTM (Boston Scientific, Marlborough, MA, USA). Additional post-dilatation was done with the kissing-balloons technique - balloon catheters $3.0 \times 28 \mathrm{~mm}(\mathrm{LAD})$ and $2.0 \times$ $15 \mathrm{~mm}$ (Dg2) (Figure $1 \mathrm{~B} \mathrm{~d}$ ). The final effect was confirmed in IVUS (Figure 2 B). Afterwards, the Dg2 guide wire (Figure $1 \mathrm{~B}$ d) was removed and inserted into the circumflex (CX) branch (Figure $1 \mathrm{~B}$ a) for final post-dilatation with the kissing-balloon technique of LMCA/LAD/CX. Thereafter we deployed directly DES Xience PRO ${ }^{\mathrm{x}} 4.0 \times 33 \mathrm{~mm}$ in the LMCA and proximal LAD. Subsequently post-dilatation was performed with the non-compliant balloon catheter NC Quantum Apex (Boston Scientific, Marlborough, MA, USA) (Figure $1 \mathrm{C}$ ). The overlapping zone (Figure $1 \mathrm{~B} \mathrm{C}$ ) was located a few millimeters distally to Dg1 (Figure $1 \mathrm{~B}$ b) and proximally to Dg2 (Figure 1 B d). Unfortunately, during removal of the protective guide wire from Dg1 fracture and separation of the distal part of the guide wire occurred (Figure $1 \mathrm{D}$ ). The retrieval of the separated guide wire was performed using an Amplatz GooseNeck Snare Kit and then a Microsnare Kit catheter (Figures $1 \mathrm{E}$ and 3). Unfortunately, the control IVUS revealed that the distal part of the stent implanted in the LAD was rolled up by the trapped guide wire during retrieval (Figure $2 \mathrm{C}$ ).

\section{Corresponding author:}

Stanisław Bartuś MD, PhD, 2 ${ }^{\text {nd }}$ Department of Cardiology, University Hospital, 17 Kopernika St, 31-501 Krakow, Poland, phone: +48 501253 853, e-mail: sbartus@cathlab.krakow.pl

Received: 19.08.2015, accepted: 7.10.2015. 

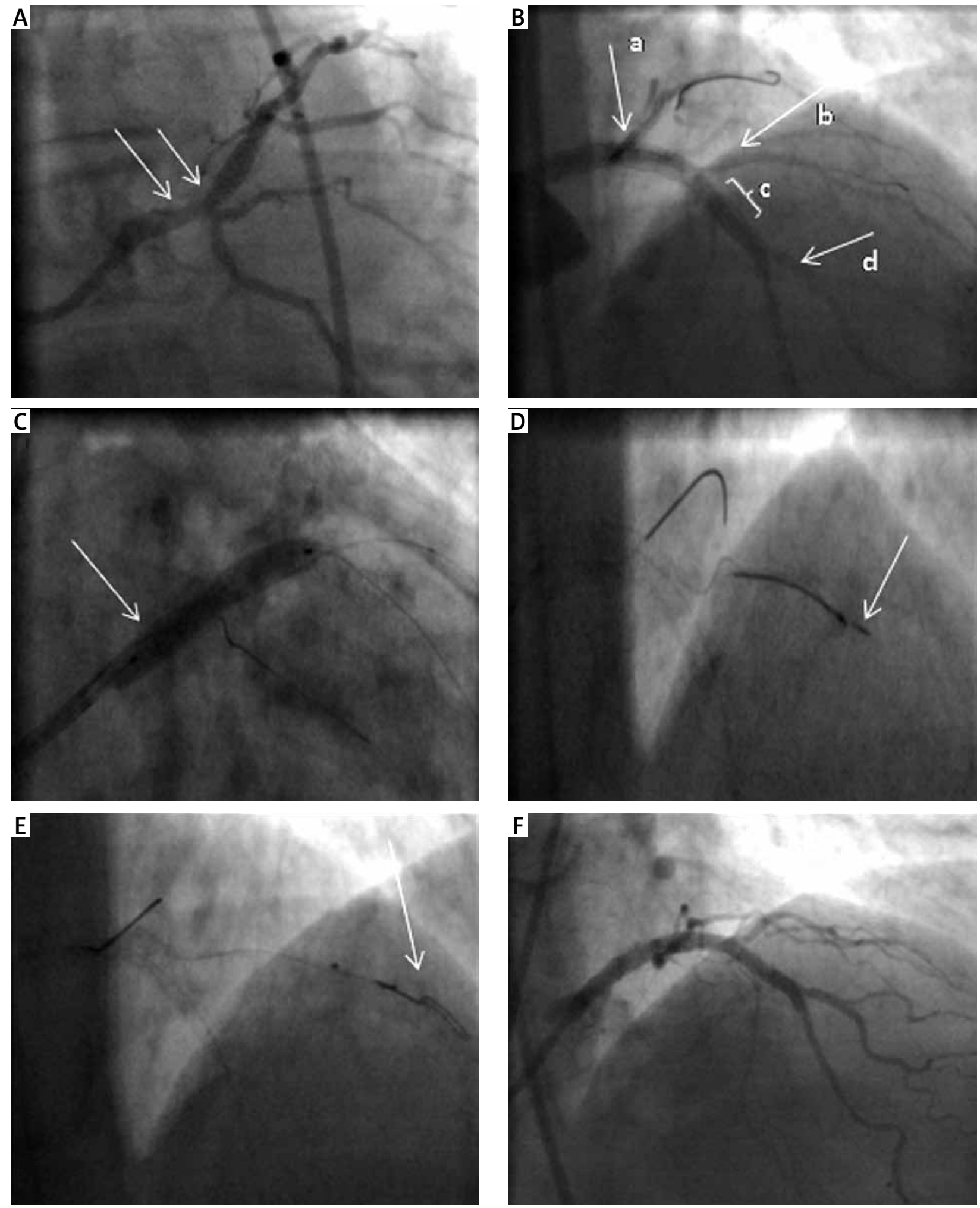

Figure 1. Fluoroscopic images of coronary arteries. A - Cine image in left anterior oblique caudal (LAO) view of the left system angiogram showing significant disease in LAD and LMCA (marked with arrow). B - Cine image in right anterior oblique (RAO) cranial view of the stent deployment in the LAD (a - circumflex branch with guide wire; $b$ - first diagonal branch with guide wire; $c$ - overlapping stents zone; $d$ - second diagonal branch after post-dilatation with kissing-balloons technique). C - Cine image in RAO cranial view of the stent deployment in the LMCA/LAD (marked with arrow). D - Cine image in RAO cranial view of the broken guide wire (marked with arrow). E - Cine image in RAO cranial view of the Amplatz GooseNeck Snare Kit Catheter with the broken guide wire (marked with arrow). F - Cine image in RAO cranial view of the final result 
We first decided to perform in-stent balloon angioplasty. However, our efforts were not efficient. Subsequently, we decided to implant two more DES in the distal part of the previously implanted stent to LAD Xience PRO $\times 3.5 \times 12 \mathrm{~mm}$ and in the proximal Xience PRO ${ }^{\times} 4.0 \times 12 \mathrm{~mm}$. The final optimal result of $\mathrm{PCl}$ was confirmed through angiography and IVUS (Figures $1 \mathrm{~F}$ and $2 \mathrm{D}$ ).

\section{Discussion}

Excessive torquing and bending, forceful withdrawal of a catheter or stent which is entrapped due to arterial spasm, inappropriate handling of a catheter, reuse, manufacturing flaws, inadvertent passage of a large catheter through smaller access site sheaths, polymer aging or a combination of mentioned factors could be the cause of catheter fracture [2, 3]. Additionally, too many protective guide wires located in small vessels could increase the risk of wire entrapment. Therefore, a conservative estimate of the need to protect non-essential branches should be performed prior to the endovascular procedure. Nowadays, the leading opinion is that broken wires and catheters need to be removed, because the remnants could be a nidus for vessel wall injury, thrombosis, infarctions or arrhythmias. In some cases such complications could be life-threatening and conversion to open cardiac surgery is inevitable. A recently published large literature review concerning the management of retained intervention guide-wires revealed that among 67 included patients surgical extraction was performed in 29 (43.3\%) cases, percutaneous therapy was used in $28(41.8 \%)$ cases, and in $10(14.9 \%)$ cases conservative therapy was applied [4]. Noteworthy is that floppy wires were used in most cases [4]. Nevertheless, endovascular removal is easier, faster, more elegant and usually less disabling for the patient. Cardiac surgery in urgent mode is associated with significant morbidity and mortality, and consists of bypass surgery, direct coronary arteriotomy or aortotomy. There are many available devices and techniques which can be used for percutaneous retrieval of broken wires and catheters including stenting against the vessel wall, snare loop, double or triple wire techniques, bioptome, Tornus micro-catheter, deep-guide wire catheter wedging with balloon inflation and pigtail catheter [4, 5]. Vascular or stent complications are usually most efficiently diagnosed by IVUS [6]. Failure of the retrieval procedure may result in ischemia due to coronary thrombosis, new fragmentation, coronary spasm, obstruction or arterial dissection and rupture complicated by tamponade and cardiac surgery [4]. In some cases operators decide to leave the fractured part of the wire in the artery, which is pro-thrombotic. In
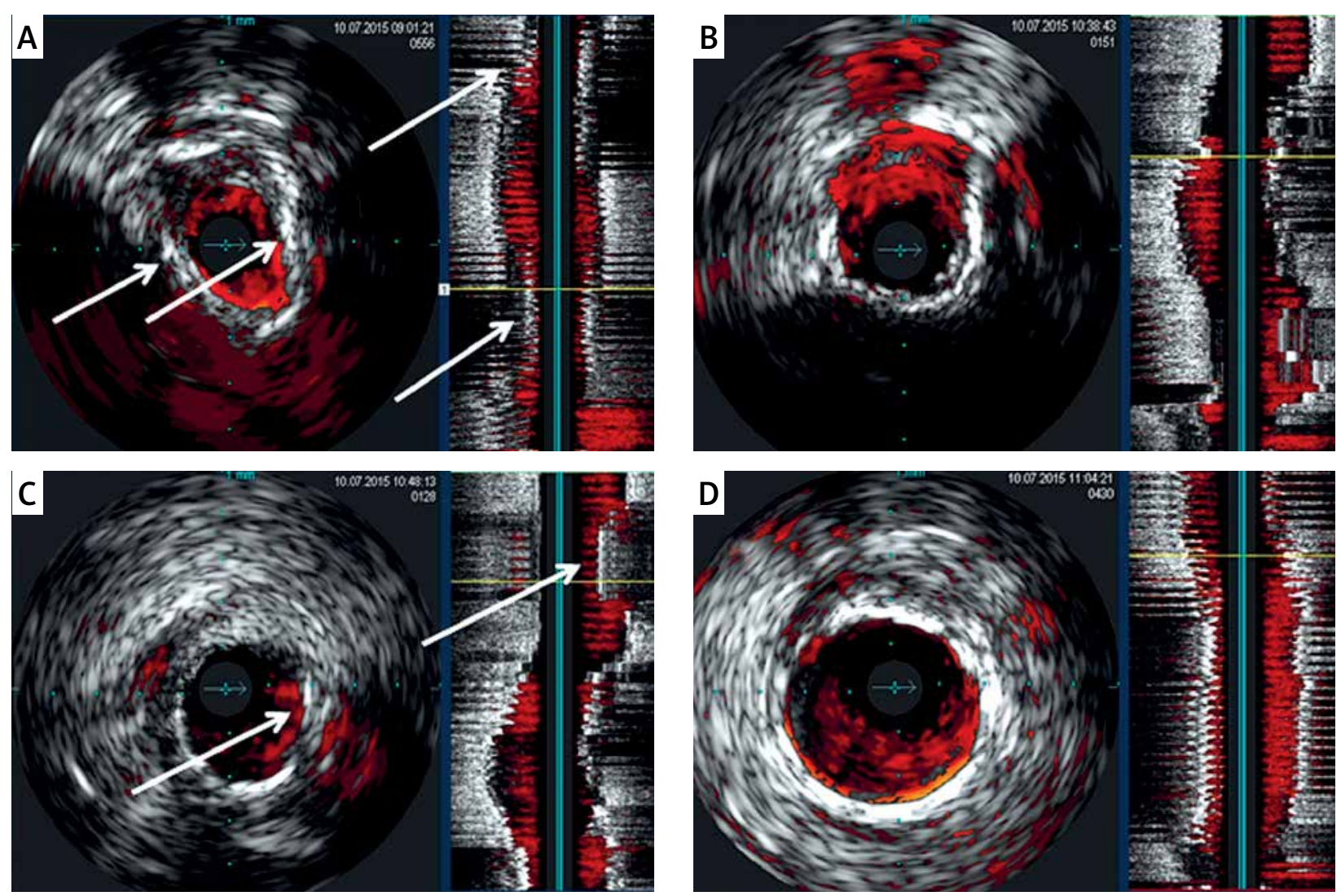

Figure 2. Intravascular ultrasound captures: A - LAD stenosis before PCl (marked with arrows). B - After PCI of LMCA/LAD. C - Stent deformation in LAD (marked with arrows). D - Final picture after deployment of two additional stents 

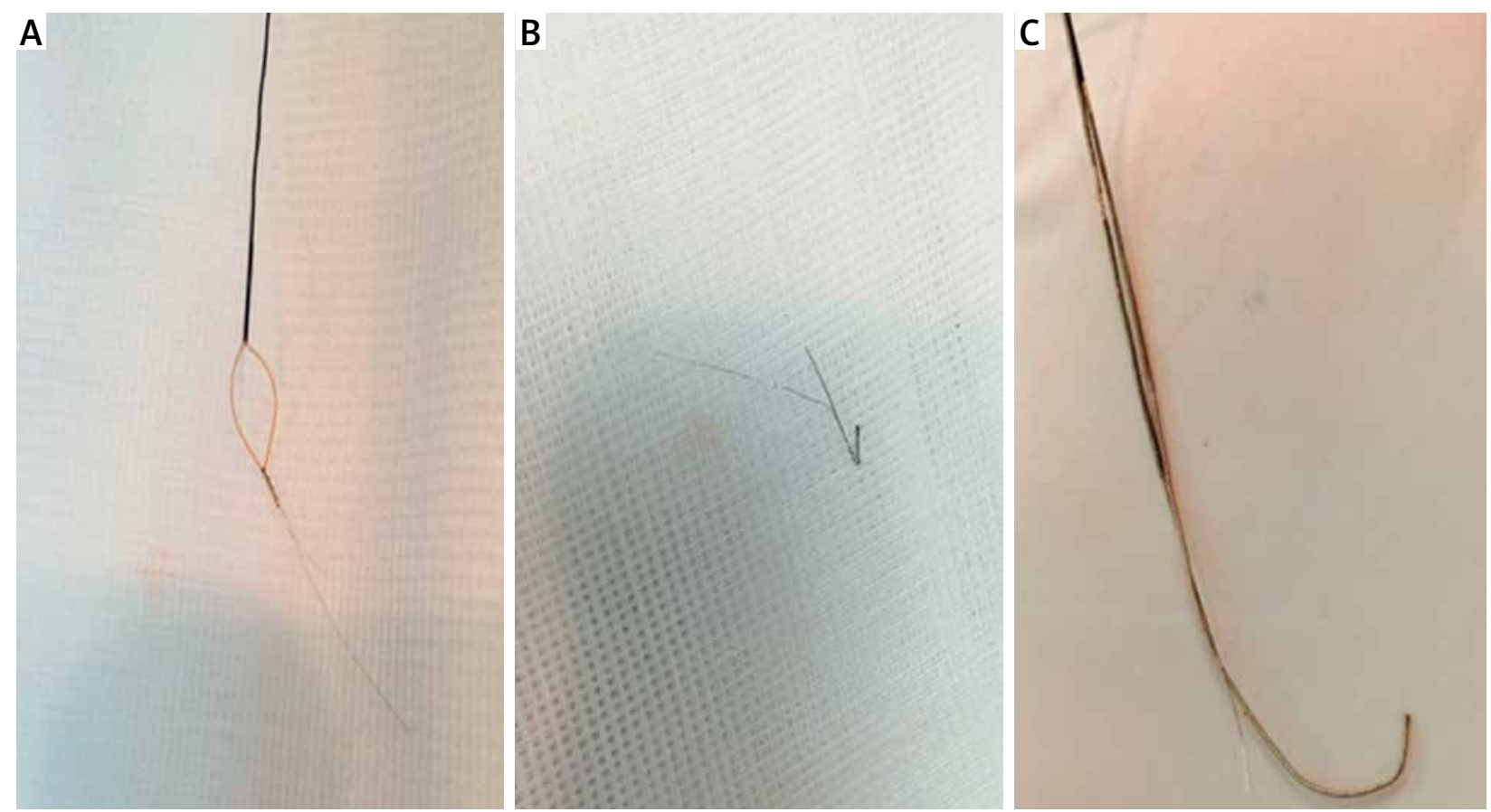

Figure 3. Photographic images: A - The Amplatz GooseNeck Snare Kit Catheter with the broken guide wire. B - The broken distal part of the guide wire. C - The Amplatz GooseNeck Snare Kit Catheter

the current case, the Amplatz GooseNeck snare catheter was used for removal of the broken guide wire, and its effectiveness and usefulness have been demonstrated in numerous studies [7, 8]. Unfortunately, the retrieval procedure was complicated by partial rolling up of the stent deployed in the LAD. Ballooning of the rolled stent did not bring a satisfactory effect, so we decided to place additional stents. This raises the question: is it worth removing the wire trapped by the stent? The rate of stent thrombosis in patients after $\mathrm{PCl}$ with DES implantation ranges from $1.4 \%$ to $1.8 \%$ in 3-year follow-up [9], while the risk of thrombosis in artificially deformed stents is not supported by evidence-based medicine. In selected patients it seems to be reasonable to leave the guide-wire in situ. Such treatment may be justified in patients with distally occluded or small vessels [4]. These individuals require close follow-up and urgent surgical treatment if ischemic events occur. Answering this question would be possible after completion of an analysis on a large group of patients. In the case of a watch-and-wait approach, the personal risk of thrombosis and hemorrhagic complications must be estimated. Additionally, this way usually requires control angiography in the future and more intensive and prolonged antiplatelet therapy. In the current case we decided to perform $\mathrm{PCl}$ with stent implantations in the place of the deformed stent. Based on our experience, this procedure is safer and more comfortable for the patient.

\section{Conclusions}

We confirmed the usefulness of the Amplatz GooseNeck snare catheter in the hands of an experienced in- terventional cardiologist and in specific circumstances. However, the way of further treatment necessitated by artificial deformation of the previously implanted stent after the retrieval procedure is still under discussion and should be investigated in further studies. Undoubtedly, this type of retrieval procedure should be performed with the background of cardiovascular surgery team. Moreover, it has to be considered that implantation of subsequent stents and increase of their number and length bring increased risk of thrombosis and restenosis. And finally, the risk of guide wire disruption could be decreased by minimizing the number of guide wires in use, minimal manipulation of the guide wire, and by removing side branch wires before left main stenting.

\section{Conflict of interest}

The authors declare no conflict of interest.

\section{References}

1. Hartzler GO, Rutheford BD, McConahay DR. Retained percutaneous transluminal coronary angioplasty equipment components and their management. Am J Cardiol 1987; 60: 1260-4.

2. Schneider RM, Fornes RE, Stuckey WC, et al. Fracture of a polyurethane cardiac catheter in the aortic arch: a complication related to polymer aging. Cathet Cardiovasc Diagn 1983; 9: $197-$ 207.

3. Macovei LG, Presura RM, Anghel L, et al. Coronary stent entrapment. Postep Kardiol Inter 2014; 10: 216-8.

4. Al-Moghairi AM, Al-Amri HS. Management of retained intervention guide-wire: a literature review. Curr Cardiol Rev 2013; 9: 260-6. 
5. Hawranek M, Desperak PD, Gasior PM, et al. Double stent loss during multivessel percutaneous coronary intervention in nonST-segment elevation acute coronary syndrome. Postep Kardiol Inter 2014; 10: 114-8.

6. Tyczyński P, Chmielak Z, Pręgowski J, et al. Intervention on the left main coronary artery. Importance of periprocedural and follow-up intravascular ultrasonography guidance. Postep Kardiol Inter 2014; 10: 130-2.

7. Yedlicka JW Jr, Carlson JE, Hunter DW, et al. Nitinol gooseneck snare for removal of foreign bodies: experimental study and clinical evaluation. Radiology 1991; 178: 691-3.

8. Bartuś S, Kameczura T, Chyrchel M, et al. Rescue removal of disrupted balloon catheter from right coronary artery and aortic arch. Kardiol Pol 2013; 71: 772-4.

9. Camenzind E, Wijns W, Mauri L, et al. Stent thrombosis and major clinical events at 3 years after zotarolimus-eluting or sirolimus-eluting coronary stent implantation: a randomised, multicentre, open-label, controlled trial. Lancet 2012; 380: 1396-405. 